\title{
Reducing Language Barriers and Racial/Ethnic Disparities in Health Care: An Investment in Our Future
}

\author{
Somnath Saha, $M D, M P H^{1,2}$, Alicia Fernandez, $M D^{3}$, and Eliseo Perez-Stable, $M D^{3}$ \\ 'Section of General Internal Medicine, Portland VA Medical Center (P3MED), Portland, OR, USA; ${ }^{2}$ Division of General Internal Medicine \& \\ Geriatrics, Oregon Health \& Science University, Portland, OR, USA; ${ }^{3}$ Department of Medicine, University of California San Francisco, San \\ Francisco, CA, USA.
}

KEY WORDS: language; ethnic groups; delivery of health care. J Gen Intern Med 22(Suppl 2):371-2

DOI: $10.1007 / \mathrm{s} 11606-007-0372-4$

(c) Society of General Internal Medicine 2007

I n 2003, the Institute of Medicine issued a groundbreaking report that shone a spotlight on the problem of racial and ethnic disparities in health care in the United States. ${ }^{1}$ It is now well recognized that minority Americans tend to receive less care, and lower-quality care, than the majority (white) population. The reasons behind this phenomenon are complex and opaque. There is no single explanation, and in many cases, we do not have an explanation. However, for millions of minority Americans, most of them foreign-born, one explanation is readily apparent: they do not speak, read, or write English, and health care in this country, with few exceptions, is delivered in English.

Two articles in this JGIM Supplement attest to the role of language barriers in explaining racial/ethnic disparities in health care. Cheng and colleagues examined the use of basic, evidence-based health care services for prevention and chronic disease management among Latinos and whites in the United States. ${ }^{2}$ They found that whites were more likely than Latinos as a whole to receive all recommended services for which they were eligible. However, when grouped by language use, Latinos who spoke English at home were not significantly less likely than whites to receive recommended services, whereas those who spoke Spanish at home were, even after accounting for education, insurance coverage, usual source of care, and a host of other potential explanatory factors. Thus, the bulk of the racial/ethnic disparity between whites and Latinos, in this case, is actually a "language" disparity, between English-speakers and Spanish-speakers.

The study by Sentell et al. produced similar findings. ${ }^{3}$ They examined access to mental health care in California, the state with the highest proportion of limited English-proficient (LEP) persons in the country. ${ }^{4}$ They found that among individuals stating a need for mental health services, Latinos were less likely than whites to have received them. However, Englishspeaking Latinos were similar to whites in their access to mental health care, whereas non-English-speaking Latinos had a markedly lower likelihood of receiving services (even when they had mental health insurance coverage). Again, the disparity here is not so much a racial/ethnic one per se, as one related to English proficiency.
The findings of these studies indicate not only that language plays a large role in explaining racial/ethnic disparities, but that comparing groups by race/ethnicity alone may substantially underestimate the degree of inequity between the haves and the have-nots. For instance, in the study by Sentell et al., Latinos as a whole were about one-third less likely to receive needed mental health services when compared to the overall California population ( $28 \%$ vs $45 \%$ ). But Latinos who spoke no English were $85 \%$ less likely to receive services compared to the general, English-speaking population (8\% vs $51 \%$ ). ${ }^{3}$

In the same vein, examining groups by English proficiency can reveal "hidden" disparities. Sentell and colleagues found that Asians (whom we often do not think of as a disadvantaged group) did not have significantly lower access to mental health care than whites, after accounting for other factors. However, splitting the Asian population, into those who spoke English and those who did not, revealed stark disparities. NonEnglish-speaking Asians were substantially less likely than the general, English-speaking population to receive needed services (11\% vs $51 \%$ ). ${ }^{3}$ Clearly, focusing on Latinos and Asians as racial/ethnic groups, without attention to subgroups defined by English proficiency, carries the potential of overlooking large inequities in health care.

It must be acknowledged that the inequities experienced by LEP Americans may not be caused solely by language barriers. In the study by Cheng et al., Latinos who spoke Spanish at home were less likely than whites to receive recommended care. However, this disparity was evident when comparing whites to both LEP Latinos and to those who spoke Spanish at home but were also comfortable conversing in English $^{2}$; i.e., Spanish-speaking patients received fewer services, even when they could speak to their doctors in English. This finding suggests that factors other than language itself may play a role in the disparities observed across language groups. It is likely that language use in this instance serves as a partial proxy for acculturation, the degree to which people from other countries and cultures have adopted the norms of the majority population. Those who have not assimilated as fully to the dominant American culture may, for numerous reasons, experience lower levels of access, utilization, and quality of care.

Whatever the underlying reasons, non-English-speaking Americans are clearly a disadvantaged population when it comes to health care. Whereas numerous studies have shown that English-speaking racial and ethnic minority populationsparticularly African Americans-suffer inequities, ${ }^{1}$ LEP minorities are among the groups we treat most unfairly. This has direct relevance to health policy. If we want to remedy racial and ethnic disparities in health care, attending to language 
barriers is basically "low-hanging fruit." It targets a welldocumented source of disparities and a population experiencing dramatic inequities.

To play devil's advocate for a moment, we might ask why we should even care about disadvantages experienced by LEP populations (a question we believe exists in the minds of many people in this country, even if they do not voice it explicitly). The vast majority of Americans believe that English should be the official language of the United States. ${ }^{5}$ Racial and ethnic groups cannot change their status as minorities, but LEP Americans, by becoming English-proficient, can. So should the health care system change to accommodate LEP patients, or should LEP learn English to use the patients system health care effectively?

Such English-only rhetoric can be, superficially, quite convincing. But scratch the surface, and the arguments begin to fall apart. The non-English-speaking population of the United States has grown substantially over time. ${ }^{4}$ Although we seem loathe to admit it, we depend heavily on immigrant labor. ${ }^{6}$ And, whereas we expect immigrants to learn English, we do not have a coordinated system for making that happen. Likewise, whereas we say that we want them to integrate into our society, and assimilate into the mainstream U.S. culture, we also tend not to want them moving into our communities. ${ }^{7}$ As our former Surgeon General, Richard Carmona, attests in his foreword to this Supplement, we push LEP immigrants to the fringes of our society. ${ }^{8}$ The net effect is that many immigrant groups form communities of their own, in which they are able to go about their daily lives in their native languages...until they get sick.

There are many reasons-ethical, legal, and practical-that it is incumbent on us to better serve the health needs of LEP populations. We are a nation founded on the tenets of equality, justice, and unity. It goes against our founding principles to tolerate the inequities that the studies in this Supplement, and many others, reveal. Moreover, it goes against our laws. As Chen et al. ${ }^{9}$ have reiterated, not attending to language barriers amounts to discrimination on the basis of nationality, a violation of our cherished Civil Rights Act. Finally, it is in our best interest to adequately care for LEP populations and other minority groups, to protect the diversity that is one of our greatest economic and cultural assets. This country was built by immigrants (voluntary and forced); its foundation is made of a concrete formed by the mixing of diverse values and cultures. In today's global economy, our multicultural and multilingual environment is more important than ever, providing an advantage shared by few other nations. It would be foolish not to nurture this advantage by providing good health care across cultures and languages.

Achieving this goal, however, is easier said than done. The studies in this Supplement provide hopeful advances, but we still have much to learn about how to best address language barriers. As Partida ${ }^{10}$ points out, we will need coordinated, systemic efforts to conduct the research and develop the solutions needed to transform our English-language health care system into one that is fully accessible to LEP Americans.
The foreign-born population of the United States is expected to grow for decades to come, ${ }^{11}$ and accordingly, our population will remain multilingual for the foreseeable future. Transforming our health care system to deliver care effectively across language barriers is an essential investment in that future.

Acknowledgments: Dr. Saha is supported by an Advanced Research Career Development award from the Department of Veterans Affairs, Health Services Research \& Development Service, and by a Generalist Physician Faculty Scholars award from the Robert Wood Johnson Foundation. The opinions expressed in this editorial are those of the authors and not necessarily those of the Department of Veterans Affairs or the Robert Wood Johnson Foundation.

Corresponding Author: Somnath Saha, MD, MPH; Section of General Internal Medicine, Portland VA Medical Center (P3MED), 3710 SW U.S. Veterans Hospital Rd., Portland, OR 97239, USA (email: sahas@ohsu.edu).

\section{REFERENCES}

1. Smedley BD, Stith AY, Nelson AR, eds. Unequal Treatment: Confronting Racial and Ethnic Disparities in Health Care. Washington, DC: National Academies Press, 2003.

2. Cheng EM, Chen A, Cunningham w. Primary language and receipt of recommended health care among Hispanics in the United States. J Gen Intern Med. 2007 (in press) DOI 10.1007/s11606-007-0346-6.

3. Sentell T, Shumway M, Snowden L. Access to mental health treatment by English language proficiency and race/ethnicity. J Gen Intern Med. 2007 (in press) DOI 10.1007/s11606-007-0345-7.

4. Shin HB, Bruno R. Language Use and English Speaking Ability: 2000. Census 2000 Brief. Washington, DC: U.S. Census Bureau; October 2003.

5. Rasmussen Reports. 85\% support English as official language of U.S. Available at: http://www.rasmussenreports.com/public_content/politics/ 85_support_english_as_official_language_of_u_s. Accessed September 3, 2007.

6. Sum A, Harrington P, Khatiwada $\mathbf{I}$. New foreign immigrants and the labor market in the U.S.: the unprecedented effects of new foreign immigration on the growth of the nation's labor force and its employed population, 2000 to 2004. Boston, MA: Center for Labor Market Studies, Northeastern University; January 2005. Available at: http://www.aypf. org/publications/U.S.Immigrationpaperjanuary2005.pdf. Accessed September 3, 2007.

7. Ellen IG. Sharing America's Neighborhoods: The Prospects for Stable Racial Integration. Cambridge, MA: Harvard University Press, 2000.

8. Carmona RH. Improving language access: a personal and national agenda. J Gen Intern Med. 2007 (in press) DOI 10.1007/s11606-0070376-0.

9. Chen AH, Youdelman MK, Brooks J. Legal framework for language access: Title VI and beyond. J Gen Intern Med. 2007 (in press) DOI 10.1007/s11606-007-0366-2.

10. Partida Y. Addressing language barriers: building response capacity for a changing nation. J Gen Intern Med. 2007 (in press) DOI 10.1007/ s11606-007-0367-1.

11. U.S. Census Bureau. National Population Projections. I. Summary files: total population by race, Hispanic origin, and nativity. Available at: http://www.census.gov/population/www/projections/natsum-T5.html. Accessed September 3, 2007. 